current-carrying coil must be exactly equivalent to a system of ideal 'magnetic particles'. (3) The deductions with regard to the field of a long straight current-carrying wire as usually given.

The apparatus was constructed at the instigation of Prof. C. L. Fortescue for demonstrations to students of the Electrical Engineering Department of the Imperial College (City and Guilds College).

H. E. PARK.

Electrical Engineering Department, Imperial College,

(City and Guilds College), London, S.W.7.

1 See "Electricity and Magnetism", vol. 2, Art. 477.

\section{Production of lon Clusters by X-Rays}

AcCording to recent work $^{1}$, X-ray-induced chromatid breaks in Tradescantia microspores are due to the simultaneous occurrence of about seventeen ionizations concentrated within the narrow section of a chromatid. Such clusters of ionizations can be produced by electrons with small kinetic energy, that is, by electrons approaching the end of their path through matter. It might be of interest, then, to determine how many clusters consisting of a given number of ionizations $(n)$ are produced per unit volume and per roentgen; or, which is equivalent, what fraction of all ionizations is concentrated in such clusters. For the sake of simplicity, a 'cluster' will be arbitrarily assumed to consist of the last $n$ ionizations produced by an electron at the end of its path. Accordingly, there will be as many clusters as there are electrons producing at.least $n$ ionizations along their entire path. Also for simplicity, the following calculation will be restricted to cases in which the number $n$ is greater than 2 or 3 , but is still so small that many clusters result from every photo- or Compton-electron released by X-rays.

Consider one photo- or Compton-electron $A$, the kinetic energy $T$ of which is much greater than the energy, $E_{n}=n \varepsilon$, necessary to produce $n$ ionizations $(\varepsilon \sim 32$ ev.). The number of secondary electrons ( $\delta$-rays) with kinetic energy between $E$ and $E+d E$, which are in turn released by $A$ per unit length of its path, is approximately ${ }^{2}\left(\pi e^{4} N Z \mid T\right)\left(d E / E^{2}\right)$, where $e$ is the electron charge and $N$ the number of atoms of atomic number $Z$ per unit volume. (It is assumed here that $E$ is much greater than the binding energy of atomic electrons, an assumption that may not be accurately fulfilled so far as the $K$ electrons of atoms like carbon, nitrogen, or oxygen are concerned.) The number of clusters per unit path will then be approximately:

$\left(\pi e^{4} N Z / T\right) \int_{E_{n}}^{\infty} d E / E^{2}=\left(\pi e^{4} N Z / T\right)\left(1 / E_{n}\right)$. Conse-

quently, the energy contributed by the electron $A$ per unit path to the actual production of clusters is about $\pi e^{4} N Z / T$. On the other hand, the total energy lost by $A$ per unit path is given approximately by Bloch's formula $2\left(\pi e^{4} N Z / T\right) \log \left(T / B_{Z}\right)$, where the energy $B_{Z}=11.2 \mathrm{ev}$. is an average value of the binding energy of the atomic electrons. Therefore the fraction $(f)$ of the radiation energy actually used in producing clusters, that is, the fraction of all ionizations concentrated in clusters, will be :

$$
f \sim 1 / 2 \log \left(T / B_{Z}\right)
$$

This fraction is one of the quantities characterizing the physical action of $\mathrm{X}$-rays, which are approximately independent of the velocity of the photo- or Compton-electrons and hence of the X-ray wavelength. This same fraction is also independent of the number $n$. When formula (1) is applied to tissues of living organisms, the presence of elements with different values of $Z$ in their chemical constitution must be taken into account. When $T=10^{5} \mathrm{ev}$., evaluation of the formula for hydrogen and oxygen yields $1 / 18$ and $1 / 14$ respectively; $f \sim 1 / 15$ is probably a fair estimate of the result for tissue. Since 1 roentgen of $\mathrm{X}$-rays produces approximately 1.7 ionizations per $\mu^{3}$ tissue, it will also produce about $1.7 \mathrm{f} / \mathrm{n} \sim 1 / 10 \mathrm{n}$ clusters per $\mu^{3}$.

Because of the simplifications involved in the above calculation, its results may be regarded only as an indication of order of magnitude. The purpose was mainly to present a basically simple method, which might easily be improved upon to fit the requirements of specific applications. One rough verification will be carried out here. According to Lea and Catcheside ${ }^{1}$, the total volume of the chromatids in a Tradescantia bracteata microspore is $7 \cdot 6 \mu^{3}$, and on $\theta$ chromatid break, on the average, is produced by 20 r. X-rays. Hence one break is associated, on the average, with the production of $\sim 20 \times 7 \cdot 6 / 10 n=$ $15 / n$ clusters in the chromatids. A one-to-one correspondence of clusters and breaks may thus be obtained by taking the value $n \sim 17$ found by Lea and Catcheside. This numerical coincidence must be regarded as partly accidental, but gives support to the conclusion that chromatid breakage requires $a$ substantial number of ionizations occurring at a single time and place.

Department of Genetics,

U. FANO.

Carnegie Institution of Washington,

Cold Spring Harbor, New York. April 28.

1 Lea, D. E., and Catcheside, D. G., J. Genetics, 44, 216 (1942).

\& Bohr, N., Phil. Mag., 30, 606 (1916).

${ }^{3}$ Bloch, F., Z. Physik, 81, 363 (1933).

\section{Relation of Hypopituitarism and Starvation}

ACcording to an estimate recently made here, there are now about $400,000,000$ people in the world suffering from malnutrition. Whatever the true figures, it is apparent that a problem of appalling dimensions exists-one which is becoming increasingly a United Nations problem, and which the collapse of the enemy would throw entirely upon our hands. Assuming the best possible management of food supplies and vitamins, one wonders whether this will be enough. One also wonders whether there may not be some overlooked weapon which can be brought to bear against hunger.

After the siege of Kut in the War of 1914-18, it took the survivors, who had been on half rations for five months, from a year and a half to two years to regain their body weight and their ability to eat normal amounts of food. This great depth and persistence of effect of chronic malnutrition can be understood, I think, only in the light of some recently acquired knowledge of the endocrines. Sir Patrick Hehir during that siege observed that sometimes emaciated but otherwise unharmed soldiers were found dead in their bunks. It is likely that they died of hypopituitary cachexia. 